\title{
Calculation of Effective Molecules Collision Frequency and Cross-Sectional Ratio for Electromagnetic Discharge in Various Gases Mixtures
}

\author{
Kareem K. Mohammad \\ Department of Physics, College of Science, University of Al-Nahrain, Baghdad-Iraq. \\ E-mail: kjphy62@yahoo.com.
}

\begin{abstract}
Estimation of gases quantities in gases mixture in plasma were accomplished at $300 \mathrm{~K}$. Each mixture has an effective value of the molecular collision frequency, electro-ions collision frequency and collision cross-sectional ratio. The computational and experimental results are correlated. In this work, the concept of separate control ion energy and ion flux by the two frequencies works generally well for the investigated discharge setup. The high frequency mainly determines ion flux and radical formation in the mixture discharges while ion energies are strongly influenced by the low frequency. Deviations from an ideally separated control of ion flux and energy are caused by influences on discharge parameters and interaction between the two frequencies since the radio frequency $(\mathrm{RF})$ power sources are coupled to each other in the current setup.
\end{abstract}

Keywords: atomic and molecular transport data, molecular collisions, Boltzmann distribution, kinetic temperature, interaction of radio waves.

\section{Introduction}

For two media and low RF waves an interaction of two electromagnetic waves is observed in the ionosphere when one of them is sufficiently strong. The readable transfer of modulation is from the strong wave to the carrier of the weaker wave when strong wave amplitude is moderated. Ionosphere crossmodulation theory indicates that if the interfering waves, observed the main energy of electron increases in the region of the ionosphere, this will lead to changes in the collision frequency of electron. The required modification is the presence of the observed disturbing wave if the absorption of an electromagnetic wave in the region of ionosphere is determined by collision frequency of the electron in the region. According to the effect of earth's magnetic field the theory extended to transfer modulation at radio frequencies corresponding to the gyro-frequency of the electrons was predicted which has been observed experimentally [1-7]. In gaseous discharge plasma, controllable laboratory experiments of interaction between microwaves are simultaneously propagated. The aim of this work is to verify the experimental data with the theoretical values to study the collision processes for different percentages of Ar-He$\mathrm{N}_{2}$ and $\mathrm{Ar}-\mathrm{H}_{2}$ mixtures.

\section{Theoretical Formulation}

Assume the mechanism of energy absorption in case of wave interaction phenomena in gaseous discharge plasmas mentioned above for an isotropic medium is the electric field, E, has a time dependence, yield

$P_{L}=\int_{V} \frac{\left|E_{\circ}^{\prime}\right|^{2}}{2} \sigma_{r} d V$

where $P_{L}$ refers to the total dissipated power rate $E \sim E_{0}^{1} e^{i w t}, \sigma_{r}$ refers to the real part of the complex conductivity, $\sigma$; for a medium containing free electrons in a gas of heavier molecules and ions gives [3].

$$
\sigma=\sigma_{\text {real }}+i \sigma_{\text {imag }}=\frac{n_{e} e^{2}}{m}\left[\frac{v-i \omega}{\omega^{2}+v^{2}}\right]
$$

were $n_{e}$ represents the electron number density, $e$ and $m$ are the electronic charge and mass, respectively. $v$ represents the effective electron collision frequency. In an ionized gas according to the Maxwell distribution of electron velocities, the complex conductivity $\sigma$ is $[2,4,5,6]$

$$
\sigma=1.504 \frac{e^{2} n_{e}}{m}\left[\int_{0}^{\infty} \frac{v(u) u^{4} e^{-\mathbf{u}^{2}} d u}{\omega^{2}+v^{2}(u)}-i \omega \int_{0}^{\infty} \frac{u^{4} e^{-\mathbf{u}^{2}} d u}{\omega^{2}+v^{2}(u)}\right]
$$


Where

$$
u=\left(m / 2 K T_{e}\right)^{\frac{1}{2}} v
$$

$u$ is the electron energy, $K$ is the Boltzmann constant, $T_{e}$ is the gas temperature and $v$ is the electron velocity. Using the condition $v^{2}<<\omega^{2}$ for the high-frequency case and from equations 2 and 3 the effective electron collision frequency $v$ is:

$$
v=1.504 \int_{0}^{\infty} v(u) u^{4} e^{-\mathbf{u}^{2}} d u
$$

at $v^{2}<<\omega^{2}$, equation 1 becomes:

$$
P_{I}=\frac{1}{2} \frac{e^{2}}{m} \frac{v}{\omega^{2}}\left|E_{\circ}^{\prime}\right|^{2}
$$

The power balance of the average electron is [8]:

$$
P_{I}=d Q_{e} / d t+G v\left(Q_{e}-Q_{m}\right)
$$

where $P_{I}$ the energy per unit time, $G$ is an energy loss factor, $Q_{e}$ and $Q_{m}$ is the mean agitation energies of the electron and molecules respectively. In different concentrations of argon-helium-nitrogen mixtures and argon-hydrogen mixtures and electrons with energies below $\sim 2 \mathrm{eV}$, this is a good approximation for the probability of collision of electrons with the molecules is independent of velocity. From Eq. (5) the effective electron-molecules collision frequency yields [8]:

$$
v_{m}=\frac{4}{3} q_{m} n_{m} \bar{v}
$$

where

$q_{m}=2.15 \times 10^{7} \times E / N /\left[V_{d} \times(D / \mu)^{1 / 2}\right] \quad$ and $q_{m}$ refers to the effective cross sectional area of the molecules. $n_{m}$ is the molecular density, $N$ is the total gas density, $V_{d}$ is the electron drift velocity, $D / \mu$ is the diffusion coefficient to the electron mobility ratio, $E / N$ is the ratio of the applied electric field to the gas number density and $\bar{v}$ is: $\bar{v}=\left(8 K T_{e} / \pi m\right)^{\frac{1}{2}}$

Where

$e<u>=K T_{e}$

\section{Calculation of $v_{e i}$}

The effective electron collision frequency $\left(v_{e i}\right)$ with charged particles of the plasma is theoretically calculated using the following equation [8]:

$$
v_{e i}=A \frac{n_{i}}{T_{e}^{\frac{3}{2}}} \ln \left[\frac{B}{n_{i}^{0.5}} T_{e}\left(\frac{2 T_{e} T_{i}}{T_{e}+T_{i}}\right)^{0.5}\right]
$$

Here $A$ and $\mathrm{B}$ are constants, $T_{i}$ is the ion temperature, $n_{i}$ is the number of ions per cubic centimeter. In this work, we consider $n_{i}=n_{e}$.

\section{Decaying Plasma}

The relation for the rate of energy loss and gain for the average electron is given by $\mathrm{Eq}(7)$ which involves both electron-molecule and electron-ion collisions. For isothermal electrical discharge plasma this requires that $Q_{e}=Q_{m}$ where $Q_{m}=\frac{3}{2} K T_{g}$

at $T_{g}=T_{e}$ hence:

$Q_{e}=\frac{3}{2} K T_{e}$

Substituting equation 6 and 12 into equation 7 yields:

$$
\begin{aligned}
& d Q_{e} / d t=d\left(\frac{3}{2} K T_{e}\right) / d t=K \frac{3}{2} d T_{e} / d t \\
& =P_{I}-G v\left(Q_{e}-Q_{m}\right) \\
& =\frac{1}{2} \frac{e^{2}\left(E^{\prime}\right)^{2}}{m \omega^{2}}\left(v_{e i}+v_{e m}\right) \\
& -\left[G_{m} v_{m}\left(Q_{e}-Q_{m}\right)+G_{i} v_{i}\left(Q_{e}-Q_{i}\right)\right]
\end{aligned}
$$

Where $v=v_{e i}+v_{e m}$ is the total electron collision frequency $G=G_{i}+G_{m}$ is the total loss energy factor, $G_{i}$ has the significance for electron-ion collision as shown below: 
$\lambda=G\left(1-Q_{m} / Q_{e}\right)$

and

$G_{m}=2 m / M$

Where $M$ refers to the mass of the molecule, $Q_{i}$ refers to the ionic mean energy and was considered equal to $Q_{m}=Q_{\text {。 }}$ at all times. The difference $\left(Q_{e}-Q_{\circ}\right)$ is of the order of zero, which is:

$Q_{e}-Q_{\circ} \Rightarrow 0$

Substitute equation 17 into equation 13 yields:

$\frac{d T_{e}}{d t}=\frac{e^{2}\left(E^{\prime}\right)^{2}}{3 K m \omega^{2}}\left(v_{e i}+v_{e m}\right)$

This mean that at $\mathrm{t}=0, d T_{e} / d t$ is due directly to $\left(v_{e i}+v_{e m}\right)$ and $\left(E^{\prime}\right)^{2}$.where fore the rate of change of collisions frequency as a function of time, is as given below:

$d v_{e m} / d t=\frac{2}{3} q_{m} N_{m}(8 K / \pi m)^{\frac{1}{2}} T_{e}^{-\frac{1}{2}} d T_{e} / d t$

and as well as $v_{e i}$

$d v_{v i} / d t=$

$-\frac{3}{2} A n_{i} T_{e}^{-5 / 2}\left\{\left[\ln ^{2}\left[B T_{e} e_{i}^{-1 / 2}\left(2 T_{e} T_{i} /\left(T_{e}+T_{i}\right)^{-1}\right)^{\frac{1}{2}}\right]-1 / 3 T_{e} /\left(T_{e}+T_{i}\right)^{-1}\right\} d T_{e} / d t\right.$

\section{Experimental values of $v_{e i}$}

With $A=3.6 \quad$ and $B=3.7 \times 10^{3}, \quad n_{i}=n_{e}$, $T_{e}=T_{i}$ and by substituting these experimental values into equation 11 yields [9] :

$v_{e i}=3.6 n_{i} T_{e}^{-3 / 2} \ln \left[3.7 \times 10^{3} n_{i}^{-0.5} T_{e}^{3 / 2}\right]$

\section{Calculation of $q_{i}$}

An effective cross section area $q_{i}$ for collision of electrons with ions, may be defined as [7-15]: $v_{e i}=\frac{4}{3} q_{i} n_{i} \bar{v}$

where:

$q_{i}=\frac{3}{2} \frac{\Gamma^{e}}{u^{2}}$

$\Gamma^{e e}=e^{2} / 24 \pi \varepsilon_{0}^{2} \ln (\Lambda)$

$u=(m / 2 K T)^{1 / 2} v$

$v=v_{m} / N q_{m}$

$\Lambda=6.2 \times 10^{13} \times 2 \pi \times<u>^{1.5} / \omega$

$\omega=2 \pi v$

$v=1.495 \times 10^{8} \times 1 / V_{d}$

where $\varepsilon_{\circ}$ refers to the permittivity of free

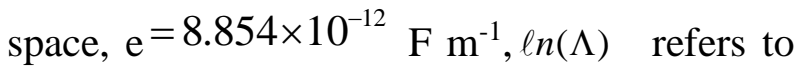
the coulomb logarithm, $\langle u>$ refers to the electron average energy, $\omega$ refers to the radial frequency and $V_{d}$ refers to the electron drift velocity. There points up the importance of electron-ion collisions, even in plasmas with low degrees of ionization.

\section{Theoretical values of $v_{\mathrm{ei}}$}

The experimental results for electron-ion collisions, as stated in equation 21 compared with theoretical works is applicable to experimental conditions. The theoretical prediction of Burckhardt, Elwert and Unsold is given by [8]:

$$
\begin{aligned}
& v_{e i}=2.25 n_{i} \times \\
& T_{e}^{-3 / 2} \ln \left[8.4 \times 10^{3} \times T_{e}^{-3 / 2} \times n_{i}^{1 / 2}\right]
\end{aligned}
$$

\section{Transport Equation Solution}

The behavior of electron interactions with gas molecules are governed by the distribution in space, energy and time of the electrons in the pure gas and/or in a mixture of gases. The prediction of the distribution function can be done by solving the electron transport equation. The general form of the transport equation may be written as $[13,14]$ : 


$$
\begin{gathered}
\frac{\partial f}{\partial t}+v \cdot \nabla_{r} f+a . \nabla v f \\
=\sum_{j} \iint\left[f(v, r, t) F_{j}\left(v_{j}, r, t\right)-f(v, r, t) F_{j}\left(v_{j}, r, t\right)\right] \\
\times v_{r j} \sigma_{j}\left(\theta, v_{r j}\right) d \Omega_{j} d v_{j}
\end{gathered}
$$

Where $\partial f / \partial t$ defines that $f(v, r, t)$ changes with time at fixed values of $v$ and $r$.

$v . \nabla_{r} f$ represents the charged particles in the vicinity of $r, a . \nabla v f$ represents the motion of the charged particles. $F_{j}$ represents the loss of electrons by collisions in $v$, and $F_{j}$ represents the gain of electrons by collision around $v . a=e E / m$ refers to the acceleration of charged particle , $F_{j}$ is the velocity distribution function of the neutral species $\mathrm{j}, v, v_{j}$ are the velocity of charged particles and the velocity of neutral species $j$ respectively, $v_{r j}=\left|v-v_{j}\right|$ refers to the relative velocity of charged particle with respect to the neutral species of gas $\mathrm{J}, \sigma_{j}\left(\theta, v_{r j}\right)$ refers to the differential microscopic cross section of the interacting charged particles with neutral species $\mathrm{J}, d \Omega_{j}$ refers to the element of solid angle.

\section{Theoretical Evaluations}

When numerically solved the transport Eq.(11), as pointed out in equation 31 gives transport coefficients, such as, electric field E, drift velocity $V_{d}$, the ratio of the coefficient diffusion to the electron mobility $D / \mu$, electron average energy $\langle u\rangle$ and electron mobility $\mu$ as shown in tables1- 4 respectively. These parameters were substituted in the calculated equations.

\section{Results and Discussion}

The value of effective collision frequency is measured for mixtures of $\operatorname{Ar}(10 \%)-\mathrm{He}(45 \%)$ $\mathrm{N}_{2}(45 \%), \quad \operatorname{Ar}(80 \%)-\mathrm{He}(10 \%)-\mathrm{N}_{2}(10 \%)$, $\operatorname{Ar}(5 \%)-\mathrm{H}_{2}(95 \%)$ and $\operatorname{Ar}(95 \%)-\mathrm{H}_{2}(5 \%) v_{\mathrm{em}}$, $v_{\mathrm{it}}, \mathrm{d} v_{\mathrm{em}} / \mathrm{dt}$ and $\mathrm{d} v_{\mathrm{ei}} / \mathrm{dt}$. These result were obtained from isothermal plasma at room temperature $(300 \mathrm{~K})$. The result, as illustrated in Fig.(1), shows the electron density and electron collision frequency as calculated from equation 8 regardless of electron-ion collision $\left(v_{e i} / v_{e m}<<1\right)$. The effective electron molecular frequency depends on total number density as $\operatorname{Ar}(10 \%)-\mathrm{He}(45 \%)-\mathrm{N}_{2}(45 \%)$ and $\operatorname{Ar}(90 \%)-\mathrm{He}(10 \%)-\mathrm{N}_{2}(10 \%)$ mixtures but in $\operatorname{Ar}(5 \%)-\mathrm{H}_{2}(95 \%)$ and $\operatorname{Ar}(95 \%)-\mathrm{H}_{2}(5 \%)$ mixtures it depends on density as frequency was found to increase with number of density. The rate of electron-molecular frequency and the electron-ion frequency with time $\mathrm{dv}_{\mathrm{em}} / \mathrm{dt}$, $\mathrm{dv}_{\mathrm{ei}} / \mathrm{dt}$ were reduced with low total collision frequency as shown in Figs. (2) and (3). On the other hand when collision frequency is equal to zero frequency rates is independent for all mixtures. This result was obtained from equation 19 and 20. The result of the electronion collision $\mathrm{V}_{\mathrm{ei}}$ as a function of electron density is shown in Fig.(4) which indicates a linear dependence on electron density $n_{e}=n_{i}$. When $v_{e i}$ is to follow the form by equation 21 . To assign a value to $B$ constant under logarithm will be difficult, because large changes in B influence the slope of $v_{e i}$ against $\mathrm{n}_{0}$. Also Fig.(a) shows that experimental values can be determined from electron-ion frequency $v_{\text {eias }}$ illustrated in equation 30 and drawn in Fig.(4) against electron density-from the above result. It shows a relation between experiment and theoretical results [6]. Finally Fig.(5) shows that electron-ion frequency dependence on gas total number density is linear as calculated from equation 22 for different gaseous mixture.

\section{Conclusions}

1. The magnitudes of effective electron molecule collision frequencies $\mathrm{V}_{\mathrm{em}}$ in a mixture of $\operatorname{Ar}(10 \%)-\mathrm{He}(45 \%)-\mathrm{N}_{2}(45 \%)$, $\operatorname{Ar}(90 \%)-\mathrm{He}(5 \%)-\mathrm{N}_{2}(5 \%), \operatorname{Ar}(5 \%)-\mathrm{H}_{2}(95 \%)$ and $\operatorname{Ar}(95 \%)-\mathrm{H}_{2}(5 \%)$ at room temperature $\left(\sim 300^{\circ} \mathrm{k}\right)$ plasma are $\left(3.320 \times 10^{7}\right.$ $\left.1.6780 \times 10^{8}\right) \mathrm{s}^{1}, \quad\left(2.689 \times 10^{7}-1.255 \times 10^{8}\right) \mathrm{s}^{1}$, $\left(1.162 \times 10^{7} 3.7330 \times 10^{7}\right) \mathrm{s}^{-1}$ and $\left(2.157 \times 10^{7}-\right.$ $\left.1.130 \times 10^{8}\right) \mathrm{s}^{-1}$ respectively. According to the above for these experiments equations 5 and 8 the effective cross sectional area of the above mixtures for electrons having average energies (0.11074-1.00852) eV, (0.37355-1.45179) eV, (0.04405-0.22271) $\mathrm{eV}$, and (0.05765-0.81091) eV respectively, may be calculated by the equation, 
$\mathrm{q}_{\mathrm{m}}=2.15 \times 10^{7} \quad \mathrm{E} / \mathrm{N} /\left(\mathrm{V}_{\mathrm{d}} \sqrt{\mathrm{D}} / \mu\right) \quad$ to be $\left(1.10108499 \times 10^{-15}-1.843 \times 10^{-17}\right) \mathrm{cm}^{2}$, $\left(2.444 \times 10^{-16}-5.788 \times 10^{-18}\right) \mathrm{cm}^{2},\left(1.700 \times 10^{-}\right.$ $\left.{ }^{14}-2.422 \times 10^{-16}\right) \mathrm{cm}^{2}$, and $\left(2.876 \times 10^{-15}\right.$ $\left.4.015 \times 10^{-17}\right) \mathrm{cm}^{2}$ respectively.

2. A magnitude of the effective electron-ions collision frequency $v_{\mathrm{ei}}$ for the isothermal plasma at room temperature have been found using equation 22for the above mixtures which are equal to $\left(4.47470928 \times 10^{9}-1.898 \times 10^{11}\right) \quad \mathrm{s}^{-1}$, $\left(5.424 \times 10^{9}-3.309 \times 10^{11}\right) \quad \mathrm{s}^{-1,} \quad\left(2.135 \times 10^{8}-\right.$ $\left.1.249 \times 10^{10}\right) \mathrm{s}^{-1}, \quad$ and $\left(1.9946 \times 10^{9}-\right.$ $\left.7.035 \times 10^{10}\right) \mathrm{s}^{-1}$ respectively.

3 . The values of the effective cross-sectional area for electron-ion collisions $\mathrm{q}_{\mathrm{i}}$ in the above mixtures are equal to $\left(1.483 \times 10^{-13}\right.$. $\left.2.085 \times 10^{-14}\right), \quad\left(4.929 \times 10^{-14}-1.525 \times 10^{-14}\right)$ $\mathrm{cm}^{2},\left(3.124 \times 10^{-13}-8.109 \times 10^{-14}\right) \mathrm{cm}^{2}$, and $\left(2.658 \times 10^{-13}-2.498 \times 10^{-14}\right) \mathrm{cm}^{2}$ respectively at constant temperature.
4. The ratio $\mathrm{q}_{\mathrm{i}} / \mathrm{q}_{\mathrm{m}}$ of the effective cross sections for electrons in case of electron-ion and electron-molecule collisions for the above mixtures are calculated and equal to $\left(134.748-1.131 \times 10^{3}\right) \mathrm{s}^{-1},(201.679$ $\left.2.635 \times 10^{3}\right) \quad \mathrm{s}^{-1}, \quad(18.372-334.794) \quad$ and $(92.417-622.188) \mathrm{s}^{-1}$ respectively.

5 . The above defined condition indicates the importance of electron-ion collisions, even in plasmas with low degrees of ionization.

6. The mixtures parameters are obtained and compared with available experimental results for electron-ion collisions, as in equation 21 with the theoretical work as in equation 30. The best and almost exact agreement with work of Ref. [7]

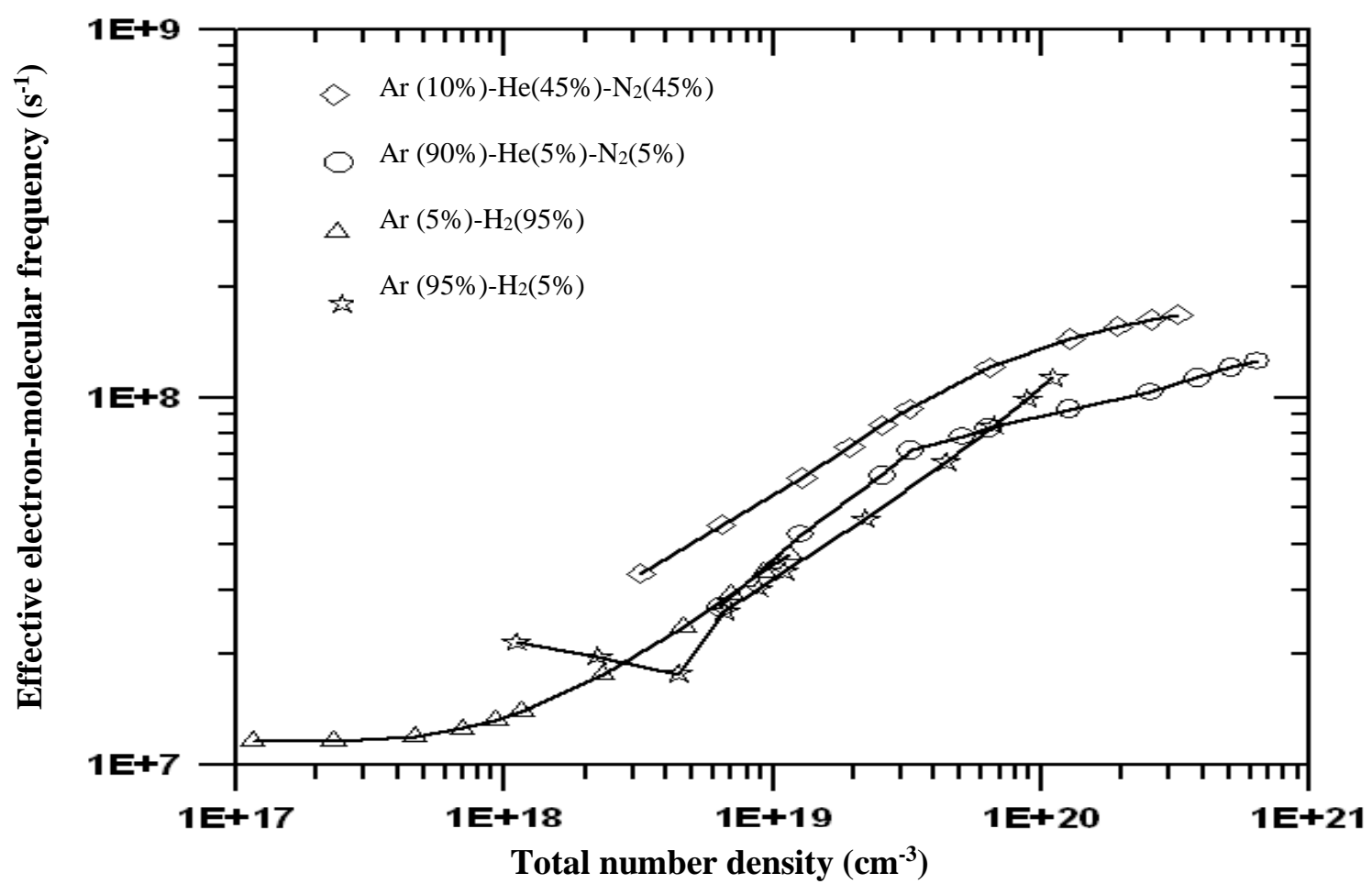

Fig. (1) The effective electron-molecular frequency, $v_{e m}$ against the gas total number density, $N$ in different mixtures. 


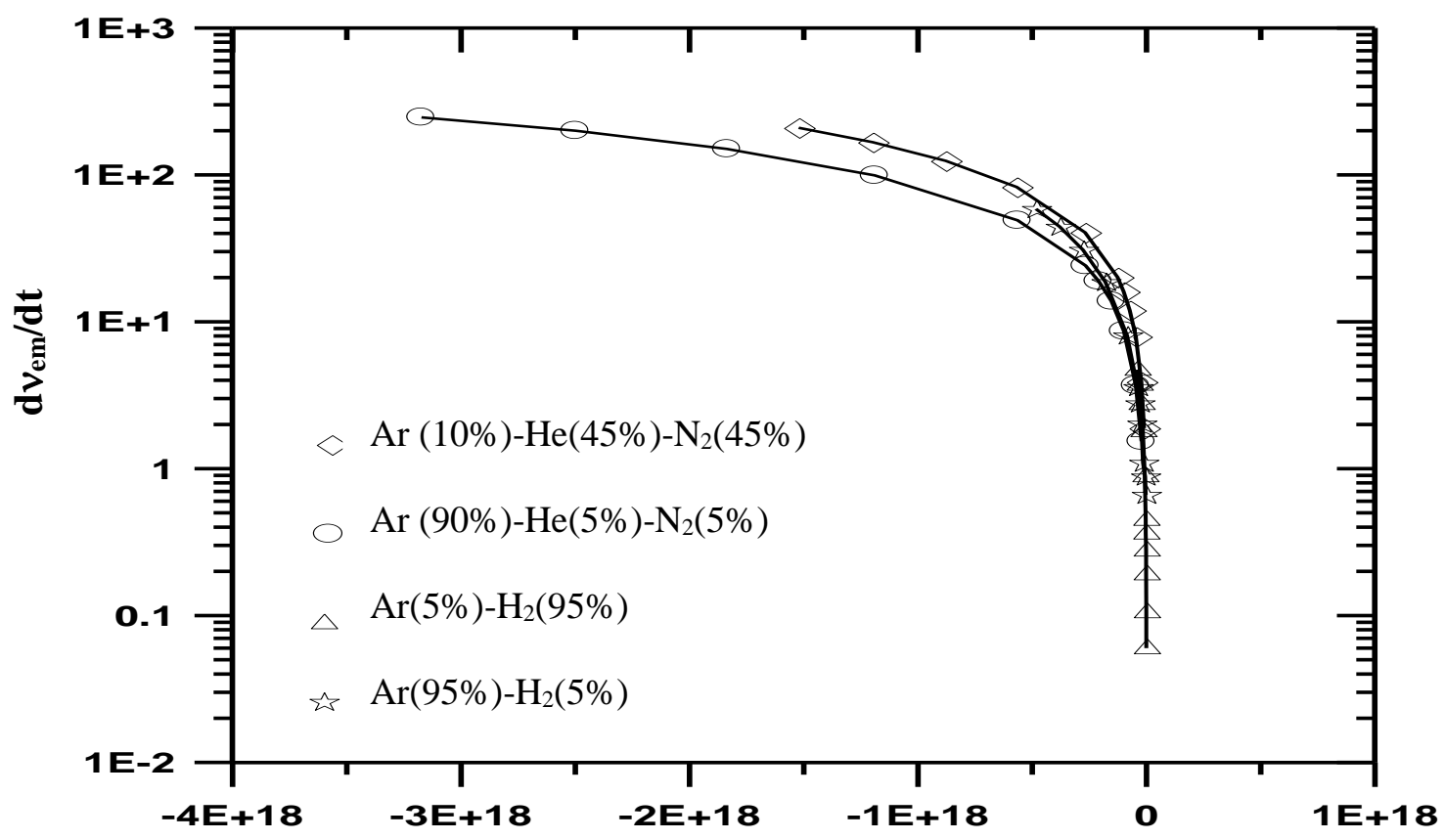

Total collision frequency $\left(\mathrm{s}^{-1}\right)$

Fig. (2) The rate of change the electron-molecular frequency with time, $d v_{e m} / d t$ against the total collision frequency, $v$ in different mixtures.

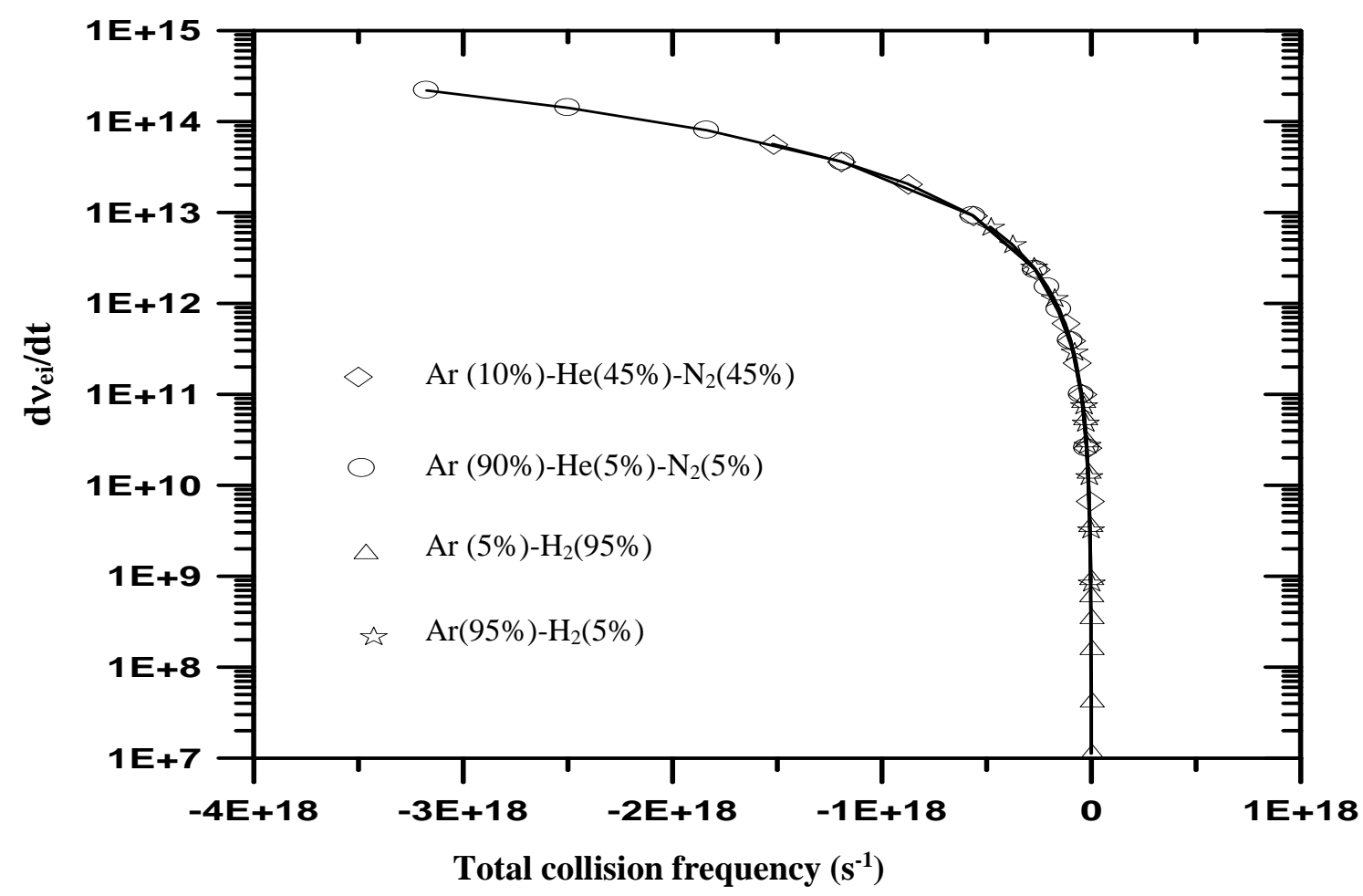

Fig. (3) The rate of change of the electron-ion frequency with time, $d v_{e i} / d t$ against the total collision frequency, $v$ in different mixtures. 


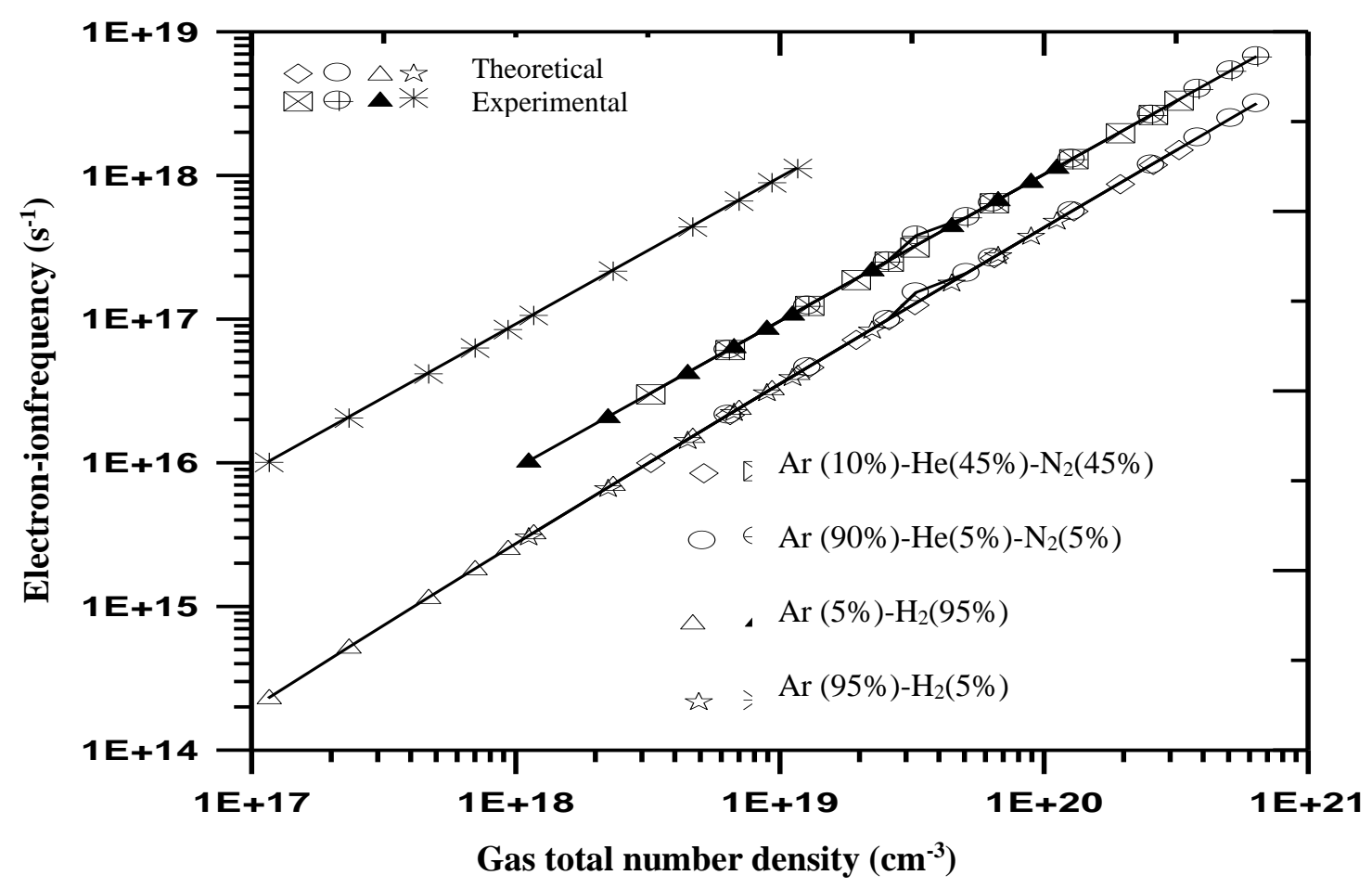

Fig. (4) The electron-ion frequency, $v_{e i}$ as a function of the gas total number density, $\mathbf{N}$ for experimental and the theoretical data in different mixtures.

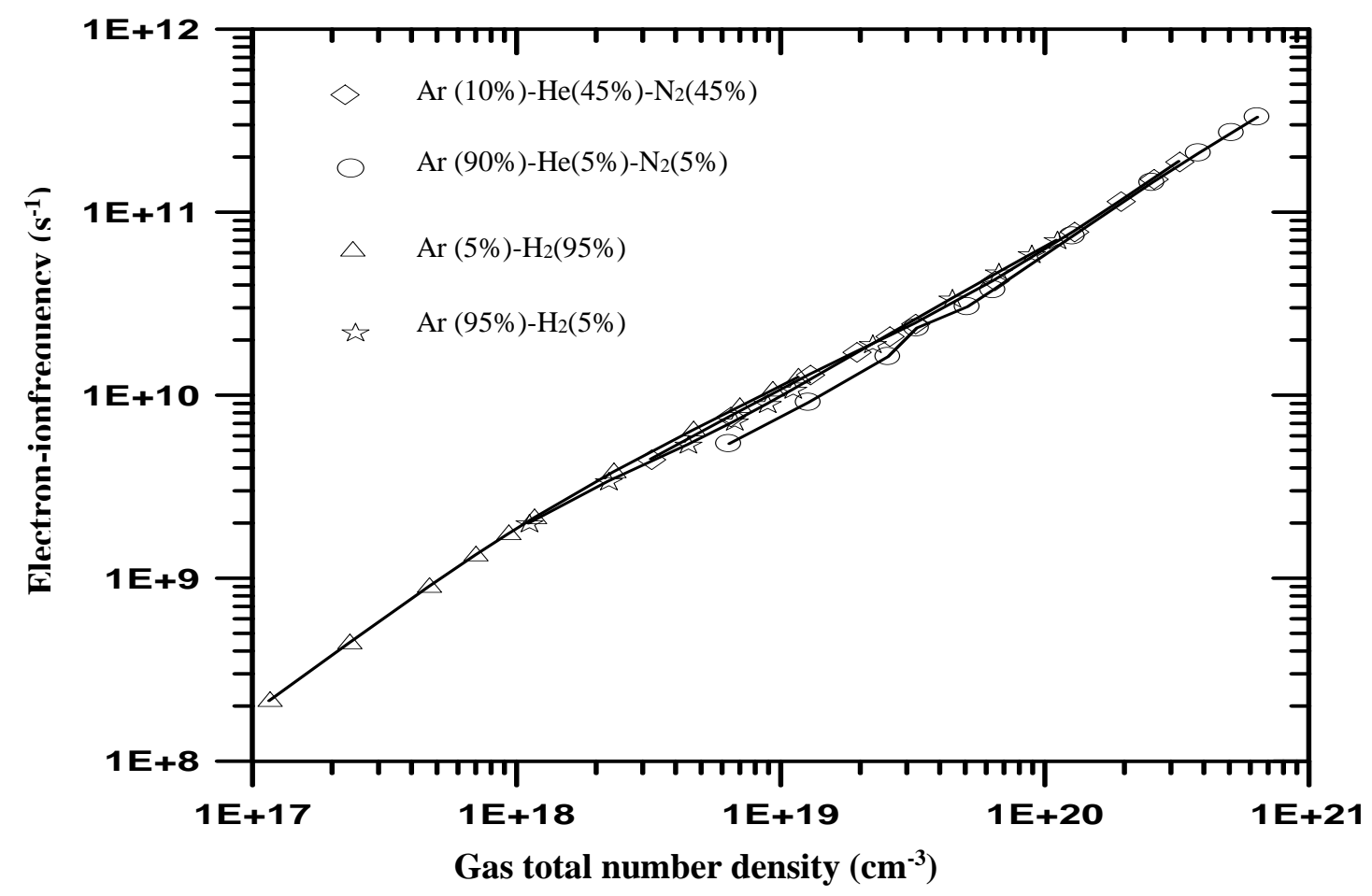

Fig. (5) The electron-ion frequency, $v_{e i}$ against the gas total number Density, $N$ in different mixtures. 
Table (1)

The calculated electron diffusion motion parameters for Argon (10\%)-Helium (45\%)Nitrogen (45\%).

\begin{tabular}{|c|c|c|c|c|}
\hline $\begin{array}{c}E \\
V \mathrm{~cm}^{-1}\end{array}$ & $\begin{array}{c}V_{d} \\
C m 5^{-1} \\
\times 10^{5} \\
\end{array}$ & $\begin{array}{l}D / \mu \\
E v\end{array}$ & $\begin{array}{l}\langle u\rangle \\
(e V)\end{array}$ & $\stackrel{\mu}{\mathrm{cm}^{2} / V . s e c}$ \\
\hline 6.426 & 1.324 & .087 & .11074 & 20610.77 \\
\hline 12.851 & 1.927 & .133 & .16471 & 14994.13 \\
\hline 25.703 & 2.890 & .199 & .25028 & 11242.15 \\
\hline 38.545 & 3.600 & .264 & .33556 & 9338.387 \\
\hline 51.406 & 4.182 & .327 & .41699 & 8136.229 \\
\hline 64.257 & 4.705 & .386 & .48879 & 7321.584 \\
\hline 128.514 & 7.061 & .597 & .71133 & 5494.023 \\
\hline 257.028 & 11.322 & .803 & .88236 & 4404.835 \\
\hline 385.542 & 15.395 & .903 & .94881 & 3993.001 \\
\hline 514.056 & 19.388 & .963 & .98433 & 3771.554 \\
\hline 642.57 & 23.289 & 1.003 & 1.00852 & 3624.391 \\
\hline
\end{tabular}

Table (2)

The calculated electron diffusion motion parameters for Argon (90\%)-Helium (5\%)Nitrogen (5\%) mixture.

\begin{tabular}{|c|c|c|c|c|}
\hline $\begin{array}{c}E \\
V \mathrm{~cm}^{-1}\end{array}$ & $\begin{array}{c}V_{d} \\
C m 5^{-1} \\
\times 10^{5} \\
\end{array}$ & $\begin{array}{c}D / \mu \\
e V\end{array}$ & $\begin{array}{l}\langle u> \\
(e V)\end{array}$ & $\begin{array}{c}\mu \\
\mathrm{cm}^{2} / V . s e c\end{array}$ \\
\hline 12.760 & 3.081 & .326 & .37355 & 24144.02 \\
\hline 25.520 & 3.677 & .551 & .56170 & 14410.29 \\
\hline 51.040 & 4.837 & .818 & $7 \overline{75098}$ & 9477.230 \\
\hline 76.560 & 6.051 & .966 & .84573 & 7904.180 \\
\hline 102.079 & $\overline{7.296}$ & 1.058 & .90095 & $\overline{c 7147.368}$ \\
\hline 127.590 & $\overline{8.568}$ & $\overline{l 1.119}$ & $\begin{array}{l}93613 \\
\end{array}$ & 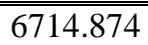 \\
\hline 255.198 & 15.032 & 1.255 & 1.01688 & 5890.370 \\
\hline 510.396 & 27.002 & 1.368 & 1.11348 & 5290.533 \\
\hline 765.594 & 37.947 & 1.438 & 1.23010 & 4956.540 \\
\hline 1020.79 & 49.143 & 1.470 & 1.34983 & 48814.239 \\
\hline 1275.99 & 60.980 & 1.484 & 1.45179 & 4778.952 \\
\hline
\end{tabular}

Table (3)

The calculated electron diffusion motion parameters for Argon (95\%)- Hydrogen (5\%) mixture.

\begin{tabular}{|c|c|c|c|c|}
\hline $\begin{array}{c}E \\
V \mathrm{~cm}^{-1}\end{array}$ & $\begin{array}{r}V_{d} \\
C m 5^{-1} \\
\times 10^{5} \\
\end{array}$ & $\begin{array}{c}D / \mu \\
e V\end{array}$ & $\begin{array}{l}\langle u\rangle \\
(e V)\end{array}$ & $\begin{array}{c}\mu \\
\mathrm{cm}^{2} / V . s e c\end{array}$ \\
\hline 2.213 & .799 & .035 & .05765 & 36082.5 \\
\hline 4.426 & $\begin{array}{ll}1.698 \\
\end{array}$ & .059 & 2.09186 & 38372.11 \\
\hline 8.852 & 2.894 & .181 & .16482 & 32693.6 \\
\hline 13.279 & 3.456 & .173 & .21846 & 26028.4 \\
\hline 17.705 & 3.868 & 218 & .25709 & 21845.4 \\
\hline 22.131 & 4.250 & 253 & .28673 & 19206.0 \\
\hline 44.262 & 5.855 & .373 & .38615 & 13228.4 \\
\hline $\bar{~} 88.524$ & 7.937 & .537 & $\overline{c .52138}$ & 8965.7 \\
\hline 132.786 & 9.356 & .666 & .62894 & 7045.9 \\
\hline 177.048 & 10.485 & .775 & .72310 & 5922.2 \\
\hline 221.310 & 11.475 & .871 & .81091 & 5176.9 \\
\hline
\end{tabular}

Table (4)

The calculated electron diffusion motion parameters for Argon (5\%)- Hydrogen (95\%) mixture.

\begin{tabular}{|c|c|c|c|c|}
\hline $\begin{array}{c}E \\
V \quad c m^{-1}\end{array}$ & $\begin{array}{c}V_{d} \\
C m 5^{-1} \\
\times 10^{5}\end{array}$ & $\begin{array}{l}D / \mu \\
e V\end{array}$ & $\begin{array}{l}\langle u\rangle \\
(e V)\end{array}$ & $\begin{array}{c}\mu \\
\mathrm{cm}^{2} / V \cdot s e c\end{array}$ \\
\hline .232 & .146 & .030 & .04405 & 6355.5 \\
\hline .463 & .290 & .031 & .4461 & 62639.2 \\
\hline .926 & 2.560 & .033 & .04667 & 60432.2 \\
\hline $\begin{array}{l}1.389 \\
\end{array}$ & .798 & .035 & 2.04970 & $\overline{57453.1}$ \\
\hline 1.852 & $\begin{array}{l}1.004 \\
\end{array}$ & .038 & 205331 & $\overline{54253.0}$ \\
\hline 2.315 & 1.184 & .041 & .05727 & 51162.5 \\
\hline $\begin{array}{l}4.63 \\
\end{array}$ & 1.837 & .059 & $\begin{array}{l}.0842 \\
\end{array}$ & 39677.5 \\
\hline 9.26 & 2.651 & .095 & .11971 & 28633.2 \\
\hline 13.89 & 3.228 & .127 & .15808 & 23239.0 \\
\hline 18.52 & 3.706 & .157 & .19276 & 20011.9 \\
\hline 32.15 & 4.150 & .183 & .22271 & 17927.2 \\
\hline
\end{tabular}

\section{References}

[1] Hideaki Matsuura, et al, "Distortion of bulk-electron distribution function and its effect on core heating in fast ignition plasma", J. of Physics: conference series, 112, 1-4, 2008.

[2] Boris M. Smirnov, "Physics of Ionized Gases. John Wiley \& Sons", Inc., New York, 2001.

[3] R.D. White, K. F. Ness, R. E. Robson, "Development of swarm Transport theory in Radio-Frequency electric and crossed electric and magnetic fields", Applied surface science 192, 26-49, 2002.

[4] S. Zhaug, Hu. Xw., Liu. MH, Luo. F., Feug, Zl., "Electromagnetic wave Attenuation in atmospheric pressure plasma", plasma science \& Technology, 902, 162-164, 2007.

[5] S. A. Maiorov, "characteristic of electron drift in the Low-pressure Gas Discharge", Bulletin of the Lebedev physics Institute, 3912, 51-56, 2012.

[6] A. P. Napartovich, I.V. Kochetov, "the value of swarm data for practical modeling of plasma devices", plasma sources science \& technology, 20,2 Article No. 025001, 2011.

[7] E. D. Xaplanteris, I. S. Filippaki, "Electron drift by of Field Gradient creates many plasma phenomena: An Attempt to distinguish the cause and the effective", J. plasma physics, 78, 165-174, part2, 2012. 
[8] J. M. Anderson and L. Goldstein, "Interaction of Electromagnetic Waves of Radio-Frequency in Isothermal Plasmas: Collision Cross Section of Helium Atoms and Ions for Electrons", Phys. Rev. 100, 4, 1037-1046, 1955.

[9] L. Goldstein, etl., "Quenching of Afterglow in Gaseous Discharge Plasmas by Low Power Microwaves", Phys. Rev. 90, 3, 486-487, 1953.

[10] Saham Z. A., Ibrahim G. Faiadh, etl., "Calculations of Complex Admittance Ratio for Helium Gas, Journal of Collage of Education", Al-Mustansiryah University, Baghdad-Iraq, 5, 327-344, 2010.

[11] G.M. Petrov, etl, "An Investigation of the Positive Column of a Cd-Ne Glow Discharge: II. Afterglow", Plasma Chemistry and Plasma Processing, 21, 2, 201-222, 2001.

[12]C. R. Hoffman, etl., "Momentum Transfer Cross Sections and Conductivity Ratios For Low-Energy Electrons in $\mathrm{He}$ ", $\mathrm{Ne}, \mathrm{Kr}$, and Xe, Phys. Rev. 178, 1, 168-174, 1969.

[13]G. Cavalleri, "Measurements of Lateral Diffusion Coefficients and First Townsend Coefficients for Electrons in Helium by an Electron-Density Sampling", Phys. Rev. 179, No. 1, 186-202, 1969.

[14] V. Puech and L. Torchin, "Collision Cross Sections and Electron Swarm Parameters in Argon", J. Phys. D: Appl. Phys. 19, 23092323, 1986.

[15]S. D. Rockwood A. E. Greene, "Numerical Solutions of The Boltzmann Transport Equation, computer Physics Communications", 19, 377-393, 1980.

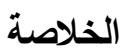

تم في هذا العمل حل معادلة الانتقال عددياً لأمزجة غازية

مختلفة بعد تعرضها لمجال كهربائي وذلك لحساب مصلحات

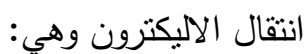

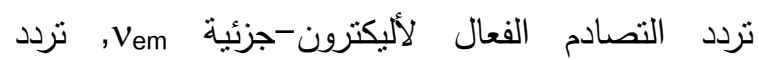

التصادم الفعال لأليكترون - أيون Vei

الفعال التصادمات اليكترون - أيون qi ونسبة المقاطع

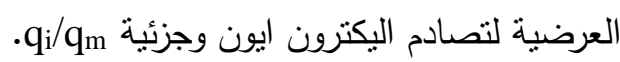

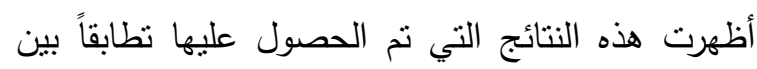

$$
\text { القيم النظرية والعملية المنشورة. }
$$

